oligen Flussigkeit eintritt. Da nun Phenol ca. 33 Procent, Kresole nach meinen Versuchen nur ca. 15 Procent Wasser ohne Trübung aufzunehmen im Stande sind, so kann man bei Unkenntmiss des Verhältnisses zwischen Phenol und Kresolen in dem Gemische der Phenole eine den Wassergehalt der abgeschiedenen Phenole berücksichtigende Correction nicht vornehmen. Diese ist auch um so weniger erforderlich, als stets auch in der concentrirtesten Salzlösung die wasserhaltigen Phenole in gewisser Menge gelöst bleiben und zwar ungefähr so viel, als Wasser von den Phenolen aufgenommen ist.

I. Beim Schütteln von $10 \mathrm{~cm}$ angeblich 100 procentiger Carbolsäure mit $10 \mathrm{ccm}$ Petroleumäther und $80 \mathrm{ccm} 10$ procentiger Natronlauge blieben ungelöst $10 \mathrm{ccm}=0$ Procent Neutralöle und Harze.

II. Beim Schiltteln von $10 \mathrm{ccm}$ angeblich 50 procentiger Carbolsäure mit $10 \mathrm{ccm}$ Petroleumäther und $80 \mathrm{ccm} 10$ procentiger Natronlauge blieben ungelöst $15 \mathrm{ccm}=50$ Procent Neutralöle und Harze.

III. Beim Schütteln von $10 \mathrm{ccm}$ angeblich $10-20$ procentiger Carbolsäure mit $10 \mathrm{ccm}$ Petroleumäther und $80 \mathrm{ccm} 10$ procentiger Natronlauge kliehen ungelöst $18 \mathrm{ccm}=-80$ Proc. Nentralöle und Harze.

Das Volumen der aus $50 \mathrm{ccm}$ der alkalischen Lösung mittelst roher Salzsäure abgeschiedenen Phenole betrug

$$
\begin{aligned}
& \text { bei } \quad I=5 \mathrm{ccm} \\
& \text { II }=2,9 \mathrm{ccm} \\
& \text { ШI = 1,1 - }
\end{aligned}
$$

woraus sich unter Zugrundelegung der Thatsache, dass die Phenole ungefähr soviel Wasser anfnehmen, als Phenole in der Salzlơsung verbleiben, der Gehalt der rohen Carbolsäure I zu 100 Procent, dej Säure II zu 49 Procent, der Säure III zu 18 Procent berechnet. Auch bei diesen Versuchen bin ich durch Herrn Dr. Sartori wesentlich unterstutzt.

\title{
Ueber die Gehaltsprüfung von Acidum carbolicum liquefactum.
}

\author{
Von H. Beckurts.
}

Nachdem Carbolsäure unter dem Namen Phenolum absolutum im Handel in Form lockerer Krystalle, welche bequem zu dispensiren sind, zu einem nicht ubermässig hohen Preise erhältlich ist, dürfte Acidum carbolicum liquefactum, welche nur ein Bequemlich- 
keitsobjekt fur die Receptur ist, als besonderes Präparat aus dem Arzneischatze wieder gestrichen werden können, zumal wir, wie meine Versuche ergaben, zur Zeit kein Mittel besitzen, die Reinheit desselben zu constatiren. Die Bestimmung des Phenols in diesem Präparate muss von dem Apotheker stets dann ausgeführt werden, wenn dasselbe aus reiner Săure nicht selbst dargestellt ist. Die zur Prufung vorgeschriebene, an zwei Maassflussigkeiten gebundene Brommethode des Arznei-Gesetzbuches ist schon hulufig als eine umständliche bezeichnet worden und an ihrer Stelle einfachere $\mathrm{Me-}$ thoden vorgeschlagen worden. Auf Grund der Erörterungen; welche in einer der vorstehenden Arbeiten gemacht wurden, bedarf die Ausführung der Brommethode, wie sie die Pharmakopoe vorgeschrieben hat, einer Modification. Zweckmässig lehnen wir uns an die schon von Schlickum zur Vermeidung des durch Verdunsten von Brom hervorgehenden Fehlers gegebenen Verhältnisse an und geben der Vorschrift die folgende Fassung:

„In jener Mischung ron $50 \mathrm{ccm}$ der Lösung, welche durch Auflösen von $1 \mathrm{~g}$ verflüssigter Carbolsăure in $1000 \mathrm{~cm}$ Wasser bereitet ist, mit je $50 \mathrm{ccm}$ der vol. Kaliumbromat - und vol. Kaliumbromidlossung, durfen nach Zusatz von $5 \mathrm{ccm}$ concentrirter Schwefelsüure und einem weiteren nach einer Frist von 10 Minuten erfolgten Zusatz einiger Krystalle von Jodkalium, zur Bindung des freigemachten Jodes $1,5 \mathrm{ccm} 1 / 10$ N.-Natriumthiosulfatlosung verbraucht worden. Sobald eine grössere Menge $1 / 10$ N.-Natriumthiosulfat verbraucht wird, ist die Carbolsäure zu schwach."

Aus der Menge der Natriumthiosulfatlősung kann man leicht die zur vollständigen Ausfällung des aus je $50 \mathrm{ccm}$ der volumetrischen Lơsungen freigemachte Broms erforderliche Mengen der Carbolsäurelosung berechnen.

$1 \mathrm{ccm} \mathrm{1/10} \mathrm{N.-} \mathrm{Natriumthiosulfat}=0,008 \mathrm{Br}=0,00156$ Phenol.

$J_{\theta} 50 \mathrm{ccm}$ beider Lősungen entwickeln durch Schwefelsäure soviel Brom als 0,0469 Phenol zu Tribromphenol zu binden vermogen.

Zieht man nun von 0,0469 die aus der verbrauchten Menge Natriumthiosulfat sich berechnende Menge Carbolsäure ab, so muss sich die Differenz zu 0,0469, wie die angewandten $50 \mathrm{ccm}$ Carbolsäurelősung zu der gesuchten überhaupt erforderlichen verhalten. Daraus berechnet sich bei dem Verbrauch von $1 \mathrm{ccm} \mathrm{1/10} \mathrm{N.} \mathrm{-} \mathrm{Natrium-}$ thiosulfatiősung 51,6 ccm Carbolsäurelösung, bei dem Verbrauche von $1,5 \mathrm{ccm}=52,6 \mathrm{ccm}$ Carbolsäurelösung. 
Unter den in Folge der Umständlichkeit des Verfahrens von verschiedenen Seiten vorgeschlagenen einfacheren Prüfungsvorschriften sind die folgenden zu erwähnen:

Hager ${ }^{1}$ empflehlt, der verflüssigten Carbolsäure durch Schütteln mit festem Chlorcalcium das Wasser zu entziehen und durch Messen der Säure vor und nach dem Schütteln mit Calciumchlorid den Wassergehalt zu bestimmen. Wie schon Vulpius ${ }^{2}$ bemerkt, hat dieses Verfahren den Nachtheil, dass die Säure durch die Wasserentziehung schon bei mittlerer Temperatur fest wird, so dass die Bestimmung nur bei hŏherer Temperatur ausgeführt werden kann. Auch ist die Menge des angewandten Chlorcalciums von Einfluss. Meine Versuche bestätigen solches.

O. Schlickum ${ }^{y}$ gründet ein Verfahren auf die Volumzunahme, welche verflüssigte Carbolsäure bei dem Schütteln mit dom gleichen Volum Wasser erfährt. Nach Angaben von Vulpius," die später von Schlickum und auch Salzer bestätigt wurden, nehmen 100 Theile reiner wasserfreier Carbolsäure ca. 36 Theile Wasser klar auf, während ein weiterer Wasserzusatz die Säure trübt. Schüttelt man nun gleiche Volumina Wasser und Phenol, so nimmt letzteres Wasser bis zur vollständigen Sättigung auf, andernfalls löst sich die vollständig gewässerte Säure auch theilweise in dem nbrigen Wasser auf, wodurch das Volum der gewässerten Carbolsäure wieder etwas vermindert wird. Nach Schlickum zeigen $10 \mathrm{ccm}$ verflüssigter Carbolsäure mit verschiedenem Wassergehalte beim Schütteln mit $10 \mathrm{ccm}$ Wasser die folgende Volumzunahme:

Verhältniss des Phenols zum Wasser. 100 Phenol. absolut. † 5 Wasser

$\begin{array}{rrrrrr}- & - & - & 6 & - & 12,45- \\ - & - & - & 7 & - & 12,3 \quad- \\ - & - & - & 8 & - & 12,1- \\ - & - & - & 9 & - & 12,0- \\ - & - & - & 10 & - & 11,85- \\ - & - & - & 11 & - & 11,7- \\ - & - & - & 12 & - & 11,6 \quad- \\ - & - & - & 13 & - & 11,5 \quad-\end{array}$

1) Hager, Commont. z. Pharm. germ. Ed. II, p. 81.

2) Pharm. Zoit. 1884, 797.

3) Pharm. Zeit. 1884, Nr. 46.

4) Pharm. Zoit. 1884, Nr. 17.
Böhe der unteren Säureschicht.

$12,6 \mathrm{ccm}$

12,45 -

12,3

$12,0-$

11,85 -

11,7 -

11,5 . 
H. Bockurts, Gehaltsprüfung von Acidum carbolicum liquefactum. 583

Verhältniss des Phonols zum Wasser. Höhe der unteren Säureschicht. 100 Phenol. absolut. + 14 Wasser

$11,+\mathrm{ccm}$

$\begin{array}{rrrr}15 & - & 11,3 & - \\ 16 & - & 11,2 & - \\ 17 & - & 11,1 & - \\ 18 & - & 11,0 & - \\ 19 & - & 10,9 & - \\ 20 & - & 10,8 & - \\ 21 & - & 10,7 & - \\ 22 & - & 10,6 & - \\ 23 & - & 10,5 & - \\ 24 & - & 10,4 & - \\ 25 & - & 10,3 & - \\ 30 & - & 9,8 & - \\ 36 & - & 9,0 & -\end{array}$

Dieses nach zahlreichen von mir ausgeführten Versuchen exacte Resultate liefernde Verfahren hat, wie zuerst Vulpius ${ }^{1}$ hervorhob, den Nachtheil, sehr kleine Volumverhältnisse zu messen, da schon Unterschiede von kaum $1 \mathrm{ccm}$ Differenzen im Wassergehalte von 8-10 Procent entsprechen. Derselbe schlug deshalb eine neue Methode vor, welche auf dem Verhalten des wasserfreien und wasserhaltigen Phenols gegen Schwefelkohlenstoff beruht. Während sich wasserfreies Phenol mit Schwefelkohlenstoff klar mischt, ruft wasserhaltige Säure darin eine milchige Trübung hervor, zu deren Aufhellung um so mehr Säure erforderlich ist, je mehr diese Wasser enthält. Bei einer Temperatur von $20^{\circ}$ bedarf es zur Wiederaufhellung der anfänglich entstandenen Trübung in $10 \mathrm{ccm}$ Schwefelkohlenstoff von_einer verflüssigten Carbolsäure:

aus 100 Theilen Phenol und 5 Theilen Wasser $1,8 \mathrm{ccm}$

\begin{tabular}{|c|c|c|c|c|c|c|}
\hline - & - & - & $-\quad 10$ & - & - & 7,0 \\
\hline - & - & - & $-\quad 12$ & - & - & 10,0 \\
\hline - & - & - & 14 & - & - & 15,0 \\
\hline - & - & - & 16 & - & - & 22,5 \\
\hline - & - & - & $-\quad 18$ & - & - & 37,5 \\
\hline - & - & - & $-\quad 20$ & - & - & 53,0 \\
\hline - & - & - & 25 & - & - & 90,0 \\
\hline
\end{tabular}

Zur Ausführung bringt man in einon verschliessbaren Messcylinder $10 \mathrm{ccm}$ Schwefelkohlenstoff und fügt zweckmässig alls einer Bü-

1) Pharm. Zoitung 1884, p. 787. 
rette so lange von der Carbolsăure hinzu, bis nach dem Umschütteln die anfangs entstandene Trübung volliger Wiederaufhellung Platz gemacht hat. Bei genauer Innehaltung einer Temperatur von $20^{\circ}$ Liefert die Methode nach meinen Yersuchen ausgezeichnete Resultate, welche man in wenigen Minuten erhält.

Eine andere Methode deutet $\nabla$ ulpius an, welche sich auf die Thatsache gründet, dass eine flüssige Carbolsäure sich mit einer um so grősseren Menge Wassers klar mischen lässt, je weniger Wasser sie bereits enthălt. Da, wie schon erwähnt, wasserfreie Carholsäure sich mit ca. 0,36 ihres Gewichtes Wasser noch blar mischen lässt, so wird man einer aus $100 \mathrm{~g}$ Phenol und $10 \mathrm{~g}$ Wasser bereiteten flüssigen Säure noch 26 Theile Wasser zusetzen können, ohne dass Trilbung erfolgt. Aus dem Volumen Wasser, das man bei Anwendung ciner bestimmten Menge Acid. carbol. liquef. bis zur bleibenden Trübung verbraucht, kann man in einfacher Weise den Wassergehalt der untersuchten Säure berechnen, da so viel Volumina Wasser, welche weniger als 26 auf 110 Vol. verflüssigter Carbolsäure zugesetzt werden durften, so viel Procent enthält letztere schon zu viel.

Th. Salzer ${ }^{1}$ führt diese Mfethode näher aus.

$100 \mathrm{~g}$ durch Wasser verflüssigte Carbolsäure im Verhältniss 100 : 10 gemischt, binden noch 23,0 Wasser

$\begin{array}{rlllll}100: 11 & - & - & - & 21,9 & - \\ 100: 12 & - & - & - & 20,8 & - \\ 100: 13 & - & - & - & 19,7 & - \\ 100: 14 & - & - & - & 18,6 & - \\ 100: 15 & - & - & - & 17,6 & - \\ 100: 16 & - & - & - & 16,6 & - \\ 100: 17 & - & - & - & 15,6 & - \\ 100: 18 & - & - & - & 14,6 & - \\ 100: 19 & - & - & - & 13,6 & - \\ 100: 20 & - & - & - & 12,7 & - \\ 100: 21 & - & - & - & 11,7 & - \\ 100: 22 & - & - & - & 10,8 & - \\ 100: 23 & - & - & - & 9,9 & - \\ 100: 24 & - & - & - & 9,0 & - \\ 100: 25 & - & - & - & 8,2 & - \\ 100: 26 & - & - & - & 7,4 & -\end{array}$

1) Pharm. Zeitung 1886, Nr. 1. 
H. Beckurts, Gehaltsprüfung von Acidum carbolicum liquefactum. 585,

\begin{tabular}{llllll}
$100: 27$ & gemischt, binden noch & \multicolumn{2}{c}{6 Wasser } \\
$1110: 28$ & - & - & - & 5,7 & - \\
$100: 29$ & - & - & - & 4,8 & - \\
$100: 30$ & - & - & - & 4,0 & - \\
$100: 31$ & - & - & - & 3,2 & - \\
$100: 32$ & - & - & - & 2,4 & - \\
$100: 33$ & - & - & - & 1,7 & - \\
$100: 34$ & - & - & - & 1,0 & -
\end{tabular}

Binden $10 \mathrm{~g}$ Acidum carbolicum liquefactum noch

0,1 Wasser, so enthalten sie 75,0 Procent Phenol

\begin{tabular}{|c|c|c|c|c|c|c|}
\hline 0,2 & - & - & - & & 75,5 & - \\
\hline 0,3 & - & - & - & - & 76,0 & - \\
\hline 0,4 & - & - & - & - & 77,0 & - \\
\hline $0, \overline{5}$ & - & - & - & - & 77,5 & - \\
\hline 0,6 & - & - & - & - & 78,5 & - \\
\hline 0,7 & - & - & . & - & 79,0 & - \\
\hline 0,8 & - & - & - & - & 80,0 & - \\
\hline 0,9 & - & - & . & - & 80,5 & - \\
\hline 1,0 & - & - & - & - & 81,5 & - \\
\hline 1,1 & - & - & - & - & 82,0 & - \\
\hline 1,2 & - & - & 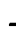 & - & 83,0 & - \\
\hline 1,3 & - & - & . & - & 83,5 & - \\
\hline 1,4 & - & - & - & - & 84,5 & - \\
\hline 1,5 & - & - & . & - & 85,0 & - \\
\hline 1,6 & - & - & - & - & 86,0 & - \\
\hline 1,7 & - & - & - & - & 86,5 & - \\
\hline 1,8 & - & - & - & - & 87,0 & - \\
\hline 1,9 & - & - & - & - & 88,0 & - \\
\hline 2,0 & - & - & - & - & 89,0 & - \\
\hline 2,1 & - & - & - & - & 89,5 & - \\
\hline 2,2 & - & - & - & - & 90,0 & - \\
\hline 2,3 & - & - & - & - & 91,0 & - \\
\hline
\end{tabular}

Zur Ausführung bringt man $10 \mathrm{~g}$ der verflüssigten Carbolsäure in einen mit Glasstöpsel verschliessbaren Cylinder, und fügt aus einer Bürette so lange Wasser hinzu, bis heim Umschütteln eine dauernd bleibende Träbung entsteht.

Aus zahlreichen Bestimmungen nach dieser Methode mögen nur zwei erwähnt werden: 
$10 \mathrm{~g}$ Ac. carbol. liquef. aus $10 \mathrm{Th}$. Phenol. absol. und 1 Theil Wasser bereitet, nahmen ohne Trübung $2,3 \mathrm{ccm}$ Wasser auf. Solche trat bei 2,4 ccm (entsprechend 91 Procent Phenol) ein.

$10 \mathrm{~g}$ Ac. carbol. liquef. aus $10 \mathrm{Th}$. Phenol. absol. und 1,5 Th. Wasser bereitet, nahmen ohne Trübung $1,8 \mathrm{~cm}$ Wasser auf, trübten sich aber bei Zusatz von 1,9 ccm entsprechend 87 Procent Phenol.

In Bestätigung der Angaben von Schlickum, Vulpius und Salzer kann ich also aus den Versuchen den Schluss ziehen, dass alle drei Methoden, namentlich aber die Schwefelkohlenstoffprobe von Vulpius und die Mischprobe Vulpius - Salzer zur Gehaltsbestimmung der verflüssigten Carbolsäure recht wohl Anwendung finden können, sobald es sich in derselben um Gemenge von reiner Carbolsäure und Wasser handelt.

Dieser Fall dürfte stets vorliegen, sobald die verflüssigte Carbolsäure in der Apotheke aus vorschriftsmässig beschaffener Carbolsäure selbst bereitet ist, und nur zur Controle des Personals dio Bestimmung ausgeführt wird. Nun hat aber schon Vulpius darauf hingewiesen, dass in den Preislisten der meisten Droguenhandlungen sich Acid. carbol. liquef. verzeichnet findet. In dieser hat man nicht allein den Gehalt an Wasser zu controliren, sondern muss auch auf einen Gehalt an den Homologen des Phenols, der Kresole etc. Bedacht nehmen.

Wie verhält sich nun eine Kresol und andere Homologe des Phenols enthaltende verflüssigte Carbolsäure gegenüber den zur Controle des Phenolgehaltes vorgeschlagenen Methoden?

Wie ich schon bei Gelegenheit der Werthbestimmung der rohen Carbolsäure ausgeführt habe, ist die Koppeschaar'sche Methode zur quantitativen Bestimmung des Phenols bei Gegenwart von Kresolen etc. unbrauchbar. Sind erhebliche Mengen der letzteren zugegen, so geben sich diese bei der Gehaltsbestimmung von Acid. carbol. liquef. durch den geringen Verbrauch von Brom zu erkennen, kleinere Verunreinigungen $(1-2 \%)$ sind dagegen nicht nachweisbar.

In einer aus $50 \mathrm{~g}$ Phenol. absol, $50 \mathrm{~g}$ Kresol und $10 \mathrm{~g}$ Wasser bereiteten Acid. carbol. liquef. ergab die Bestimmung 80 Proc. Phenol.

In einer aus $90 \mathrm{~g}$ Phenol. absol., $5 \mathrm{~g}$ Kresol und $10 \mathrm{~g}$ Wasser bereiteten Acid. carbolic. liquef. ergab die Bestimmung 86,6 Procent Phenol.

Was nun die übrigen Verfahren anbetrifft, so ergab sich bei Befolgung von Schlickum's Methode, dass das Volumen von 
II. Beckurts, Gelhaltsprïfung von Acidum carbolicum liquefactum. 587

$10 \mathrm{ccm}$ Acid. carbol. liquef., die mit Hülfe eines 50 Procent Kresol enthaltenden Phenol. abs. bereitet war, sich beim Umschwenken mit dem gleichen Volumen Wasser auf $10,5 \mathrm{ccm}$ vermehrte. Das Volumen von $10 \mathrm{~cm}$ Acid. carbol. liquef. (bereitet mit Hulfe eines 10 Procent Kresol enthaltenden Phenol. abs.) vermehrte sich beim Umschwenken mit dem gleichen Volumen Wasser auf 11,4. Das Volumen von $10 \mathrm{ccm}$ Acid. carbol. liquef. (bereitet mit Hülfe eines 5 Procont Kresol enthaltenden Phenol. absol.) vermehrte sich beim Umschutteln mit dem gleichen Volumen Wasser auf 11,5. Reine verflüssigte Carbolsäure verlangt eine Volumvermehrung auf 11,85 .

Bei Benutzung der Schwefelkohlenstoff-Methode von Vulpius bedarf es bei $20^{\circ}$ zur Wiederaufhellung der anfänglich entstandenen Trubung in je $10 \mathrm{ccm}$ Schwefelkohlenstoff

8. ccm Acid. carb. liquef. (bercitet aus 5 Proc. Kresol enth. Phenol),

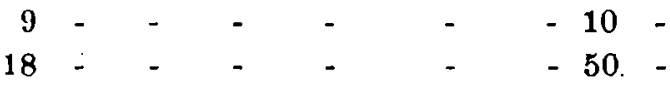

während von einer reinen verflüssigten Carbolsäure $7 \mathrm{~cm}$ erforderlich sind.

Endlich ergab die Methode von Vulpius-Salzer, dass $10 \mathrm{~g}$ Acid. carbol. liquef., bereitet aus

$50 \%$ Kresol halt. Phenol. abs., bleib. getrübt wurd. durch 1,1 ccm Wasser, 10

5 -

während $10 \mathrm{~g}$ roine verflüssigte Carbolsäure durch $2,4 \mathrm{ccm}$ geträbt wurden.

Wie diese Versuche beweisen, gestatten auch diese Methoden alle den Nachweis grösserer Mengen Kresols, nicht aber die Erkennung kleinerer Mengen desselben. Das Resultat der im Vorstehenden geschilderten Versuche ist daher dahin zusammenzufassen, dass es ausser durch die Methode Koppeschaar's auch durch die empirischen Methoden von Schlickum, Vulpius und Salzer moglich ist, zu ermitteln, ob die aus reinem Phenol dergestellte verflüssigte Carbolsäure den richtigen Gehalt an Carbolsäure besitzt, nicht aber dargethan werden kann, ob dieselbe aus reinem oder einem mit Kresolen verunreinigten Phenol dargestellt ist, da durch das vom Phenol verschiedene Verhalten der Kresole gegen Brom, Wasser, Schwefelkohlenstoff wohl grössere Mengen der letzteren, nicht aber auch kleinere Mengen sich zu erkennen geben. 
$\mathrm{Da}$ es anch an anderen Reactionen fehlt, in verflussigter Carbolsäure Verunreinigungen durch Kresole zu erkennen, aber wohl nicht ohne Grund ein sehr grosser Werth auf Verwendung einer möglichst reinen Carbolsäure gelegt wird, so erscheint der Beschluss der Pharmakopoe-Commission des deutschen Apothekervereins, Acid. carbol. liquef. als besonderes Präparat zu streichen, durchaus gerechtfertigt.

Braunschweig, im April 1886.

\section{Ueber Brandt's Schweizerpillen.}

Von Sigism. Foldhaus zu Münster in Wostfalen.

Die fortdauernden öffentlichen Anpreisungen der Brandt'schen Schweizerpillen veranlassen eine stets rege gehaltene Nachfrage nach denselben in den Apotheken. Eine Erörterung der Frage, ob die Abgabe dieser Pillen im Handverkauf in den Apotheken gesetzlich gestattet ist, erscheint deshalb von allgemeinem Interesse und so mögen die nachfolgenden Ausführungen als ein Beitrag zur Klarstellung dieser Frage hier einen Platz finden.

Far Preussen ist die Ministerial-Verfagung vom 17. August 1880, betreffend den Vertrieb von Geheimmitteln in den Apotheken, entscheidend. Nach derselben darf der Apotheker ein Mittel, das ihm in seiner Zusammensetzung nicht bekannt ist, nicht abgeben, im Falle ihm diese Kenntniss beiwohnt, selbstredend jedoch nur zu dem Preise, welchen er nach Maassgabe der Arzneitaxe zu fordern berechtigt ist. Der Verkauf der Schweizerpillen ist demnach an zwei Bedingungen geknüpft, der Kenntniss der Zusammensetzung und der Angemessenheit des Preises.

Bei dem hohen Verkaufspreise der Brandt'schen Pillen, 1 Mark für 50 abfthrende Pillen, ist eine sachliche Prüfung der Angabe uber die Zusammensetzung ohne Zweifel geboten, um so mehr, als die Herstellung der Pillen im Auslande geschieht, mithin eine amtliche Controle und Einsicht bei der Fabrikation, sowie der dabei verwendeten Substanzen ausgeschlossen ist.

In Nachstehendem soll diese Prifung in einer Weise versucht werden, wie sie in jeder A potheke mit den vorhandenen Hülfsmitteln ohne Schwierigkeit ausgeführt werden kann.

Die Pillen sollen nach Brandt's Angabe seither nach folgendem Recept angefertigt sein: 